\title{
The behavior of small helium clusters near free surfaces in tungsten
}

\author{
A. V. Barasheva, ${ }^{\mathrm{a} b}, \mathrm{H} . \mathrm{Xu}^{\mathrm{a}, \mathrm{b}^{*}}$, and R. E. Stoller ${ }^{\mathrm{a}}$ \\ ${ }^{a}$ Materials Science and Technology Division, Oak Ridge National Laboratory, Oak Ridge, TN \\ 37831-6114, USA \\ ${ }^{\mathrm{b}}$ Department of Materials Science and Engineering, University of Tennessee, Knoxville, TN \\ 37996, USA
}

\begin{abstract}
The results of a computational study of helium-vacancy clusters in tungsten are reported. A recently developed atomistic kinetic Monte Carlo method employing empirical interatomic potentials was used to investigate the behavior of clusters composed of three interstitial-helium atoms near $\{111\},\{110\}$ and $\{100\}$ free surfaces. Multiple configurations were examined and the local energy landscape was characterized to determine cluster mobility and the potential for interactions with the surface. The clusters were found to be highly mobile if far from the surface, but were attracted and bound to the surface when within a distance of a few lattice parameters. When near the surface, the clusters were transformed into an immobile configuration due to the creation of a Frenkel pair; the vacancy was incorporated into what became a $\mathrm{He}_{3}$-vacancy complex. The corresponding interstitial migrated to and became an adatom on the free surface. This process can contribute to He retention, and may be responsible for the observed deterioration of the plasma-exposed tungsten surfaces.
\end{abstract}

Keywords: plasma-facing materials; tungsten; helium clusters; molecular dynamics; kinetic Monte Carlo.

*Corresponding author. Tel.: +1-865-974-6183.

E-mail address: xhx@utk.edu (Haixuan Xu). 


\section{Introduction}

The divertors in a deuterium-tritium (DT) fusion reactor will operate at high temperatures while exposed to the high heat and charged particle fluxes arising from the DT fusion reaction. The plasma debris includes a high flux of He atoms which are a DT reaction product. Tungsten is an attractive candidate for this application due to its high melting point, low sputtering coefficient and high sputtering threshold energy [1]. A major potential impediment to successful operation of tungsten divertors is the surface erosion due to this He exposure and how He retention may influence the tritium inventory trapped in the divertor. The negative effects of the low energy $(<100 \mathrm{eV}) \mathrm{He}$ atoms implanted from the fusion plasma include nucleation and growth of $\mathrm{He}$ bubbles and surface modification due to the formation of nanorod-like structures (known as 'fuzz') [2,3]. The atomistic mechanisms responsible for these phenomena are still not well understood and are the subject of considerable experimental and computational research.

The formation, stability, and mobility of small interstitial He clusters, and the initial stages of He bubble evolution are well suited for atomistic modeling. For example, previous work in bcc metals has demonstrated that small clusters of interstitial He atoms are highly mobile, but the lattice strain associated with helium clusters containing more than a few helium atoms can lead to the formation of Frenkel pair. The vacancy is incorporated into a He-vacancy cluster which is essentially immobile at relevant temperatures, and the ejected self-interstitial atom tends to be trapped by the He-vacancy cluster [4,5]. Similar studies devoted to this subject have recently been published on the tungsten-helium system, using both density-functional theory (DFT) [4,7] and molecular dynamics (MD) [8-11]. These efforts have resulted in a rather comprehensive understanding of the relative stability of various configurations of substitutional and interstitial $\mathrm{He}$ atom in the tungsten lattice [6], and small He-vacancy complexes $\mathrm{He}_{k} \mathrm{~V}_{m}(k<6, m<5)$ [7]. This knowledge has facilitated development of new interatomic potentials for the W-He system [12], which has made it possible to perform MD modeling of the effects of helium implantation on tungsten surfaces [8-11].

Clusters containing different numbers of He atoms can be created and contribute to the radiationinduced microstructural evolution both in the bulk and near free surfaces. In this paper we present the results of atomistic simulations involving small, and thus frequently-formed, highly mobile clusters of three interstitial He atoms located near low-index $\{111\},\{110\}$ and $\{100\}$ 
free surfaces, as an initial phase of a larger investigation. It is found that the number of helium atoms required to create Frenkel pair in clusters near the surface is much smaller than that in the bulk, which indicates that surface may play an important role in helium retention. The simulations employed the self-evolving atomistic kinetic Monte Carlo (SEAKMC) method $[13,14]$. Like MD, this method describes the interatomic interactions using a set of empirical potentials; but unlike MD it does not account for atomic vibrations. This enables the simulation of much longer physical times and it has been demonstrated to provide good fidelity to the results of MD simulations $[14,15]$. We demonstrate that although small interstitial He clusters are highly mobile in bulk material far from free surfaces, there is a short-range attraction to surfaces. If trapped by the surface, the clusters reduce their energy by producing a Frenkel pair. The incorporation of the vacancy transforms the mobile interstitial He cluster into an immobile $\mathrm{He}_{3}$-vacancy complex. The corresponding interstitial migrates to and becomes an adatom on the free surface. This loss of mobility constitutes an additional mechanism for increasing He retention in tungsten, and adatom formation may contribute to the subsequent surface modification that produces tungsten fuzz at longer times. The presence of small vacancy-He clusters in the near-surface region may also increase tritium retention by trapping this otherwise mobile species.

\section{Methods}

\subsection{SEAKMC method}

We use the SEAKMC method with its constituent static relaxations and the dimer method for identifying saddle points in the present research [13-15]. The SEAKMC framework consists of several components. First, individual defects in the system are identified and the active volumes (AVs) for each defect are selected. The concept of an AV was introduced in order to increase the computational efficiency by taking into account the localized nature of most defects, and excluding distant atoms from consideration. The AVs are currently defined by a maximum distance, $r_{\mathrm{AV}}$, from any defect atom. Second, a specified number of searches, $n$, are carried out using the dimer method [16] to determine the saddle point energies for transitions between possible configurations, $E_{i}(i \leq n)$ while considering only atoms in AVs. Third, a particular transition, $i$, is randomly chosen according to the list of probabilities, $P_{i}$, weighted by corresponding Boltzmann factors, $P \propto \exp \left(-E_{i} / k_{\mathrm{B}} T\right)$, for a given temperature, $T$, where $k_{\mathrm{B}}$ is 
the Boltzmann constant. The system clock is advanced according to the residence-time algorithm [17] by $\Delta t=-\ln \xi / \sum v \exp \left(-E_{i} / k_{\mathrm{B}} T\right)$, where $\xi$ is a random number uniformly distributed on $(0,1]$, and $v$ is the attempt frequency, which is assumed here to be the same for all transitions. Fourth, depending on the nature of the defect, either local (within the corresponding AV) or global relaxation of the system is performed to move from the saddle point to another local energy minimum. This new state is the starting configuration for the next MC time step.

SEAKMC was developed to study defect evolution on the atomic level and compares well with MD when the harmonic transition state theory is applicable. Generally, it allows simulating physical processes on much longer time scales than MD, which enable the method to be used to resolve a long-standing question concerning $\langle 100\rangle$-loop formation in bcc iron [18]. In the current implementation of the model, all the saddle points found are treated as independent. This may result in some errors in the estimates of physical time, but does not affect the interaction between defects.

\subsection{Interatomic potential}

The set of interatomic potentials for the W-He system proposed by Juslin and Wirth [12] has been used. This includes a short range modification to the Ackland-Thetford W-W potential [19], a new pair-type W-He potential, and the Beck pair potential for He-He interaction [18] modified at short distances by Morishita et al. [21]. Compared with older potentials, this combination demonstrates improved agreement with the DFT formation energies of He and selfinterstitial atoms in $\mathrm{W}$ obtained in [6,7] and [22], respectively.

\subsection{Calculation details}

A box size of $20 \mathrm{a}_{0} \times 20 \mathrm{a}_{0} \times 20 \mathrm{a}_{0}$ with $\sim 18,000$ atoms was used, where $\mathrm{a}_{0}=3.1652 \AA$ is the tungsten lattice constant. Periodic boundary conditions were employed along two (denoted as $x$ and $y$ ) directions, with a free surface perpendicular to $z$ direction as shown in Fig. 1. The AV size was defined by $r_{\mathrm{AV}}=2.7 \mathrm{a}_{0}$, based on saddle point energy convergence tests of He atom diffusion in bulk tungsten. The attempt frequency is an external parameter in the treatment of SEAKMC results and was assumed to be $v=10^{12} \mathrm{~s}^{-1}$. Unlike diffusion studies, in which the absolute value and temperature dependence of the attempt frequency are very important to the results, here it is 
just a scaling factor which influences the estimated time associated with a particular sequence of events but does not alter the events themselves. Therefore, the observations reported below do not depend on the chosen value. The number of dimer searches for transitions for each active volume was $n=12$. A larger number of saddle point searches $(n=48)$ has been carried out for the diffusion of He clusters in tungsten and no significant difference was observed between the results obtained for $n=12$ or 48 . The 'atomTV' software (http://web.ornl.gov/sci/physical_sciences_directorate/mst/fusionreactor/index.shtml) was used for graphical representation and analysis of the results.

\section{Results}

\subsection{Atomic configuration of cluster of three interstitial He atoms}

In the tungsten lattice, a cluster of three interstitial He atoms forms a triangular complex with two He atoms near one face of the unit cell, and the third He atom near a perpendicular face as shown in Fig. 2. In Fig. 2, the pair of He atoms at the base are $0.013 \mathrm{a}_{\mathrm{o}}$ above the (100) plane and the third is $\sim 0.044 \mathrm{a}_{\mathrm{o}}$ to the right of the (001) plane. The habit plane of the triangle is close to a $\{102\}$. In our simulations, the triangular cluster may have different orientations relative to the free surface, with the triangle tilted such that either the single or the pair of the He atoms is closer to the surface. We studied the following configurations at different distances from the surface:

1. For the $\{110\}$ surface, two of cluster orientations were used: (i) "triangle tip up" in which a pair of He atoms is closer to the surface and (ii) "triangle tip down" in which a single He atom is closer to the surface,

2. For the $\{100\}$ surface, two orientations were used: (i) "triangle tip up" and (ii) triangle parallel to the surface, and

3. For the $\{111\}$ surface, one orientation was used: "triangle tip up".

Representative locations are shown in Fig. 1. We note that the precise shape and orientation of the relaxed three-He-atom cluster may be somewhat altered by interaction with the surface.

\subsection{Binding energy of a three He atom cluster with the surface}

The binding energies between a cluster of three interstitial He atoms and the $\{110\},\{100\}$ and $\{111\}$ free surfaces are shown in Fig. 3 a, b and c, respectively. The interaction is short ranged, 
on the order of several lattice parameters $\left(\mathrm{a}_{0}\right)$ from the surface, and rather weak, with the maximum value of the binding energy of $0.3 \mathrm{eV}$ at a distance of one an from $\{110\}$ surface. Smaller values are observed for the other surfaces. If the relaxation is started closer to the surface, Frenkel pair formation immediately produces a tungsten adatom at the surface and a $\mathrm{He}_{3}$-vacancy complex below the surface. Note that the tungsten adatom moves in the $\langle 111\rangle$ direction from the original cluster.

\subsection{Dynamic behavior of a cluster of three interstitial He atoms}

The calculations were performed for interstitial He clusters at different distances from the free surface and at temperatures of 600, 900 and 1200K. Fig. 4 shows the saddle point and total system energies for a cluster of three interstitial He atoms at different positions in the simulation box. There is no significant dependence on the cluster location except when it is very close to the surface. Analysis of the transitions associated with these saddle point energies revealed three types of events:

1. Rotation events with the activation energy of $\Delta E \sim 0.01 \mathrm{eV}$, which do not change the center of mass of the cluster,

2. migration jumps with an activation energy in the range $\Delta E=0.13-0.35 \mathrm{eV}$, and

3. creation of an immobile $\mathrm{He}_{3}$-vacancy complex and a tungsten adatom on the surface as shown in Fig. 5, releasing an energy of $\sim 3 \mathrm{eV}$.

In addition, we made the following observations:

1. The maximum distance from the surface where a $\mathrm{He}_{3}$-vacancy complex is created depends on the surface orientation:

i. For $\{110\}$ surface: at $3^{\text {rd }}$ atomic row, which is $\sqrt{2}$ an from the surface;

ii. For $\{100\}$ surface: at $3^{\text {rd }}$ atomic row, which is 1.0 a $a_{0}$ from the surface; iii. For $\{111\}$ surface: at $8^{\text {th }}$ atomic row, which is 2.0 a from the surface.

2. A cluster of three interstitial He atoms diffusing from the bulk toward the surface and reaching the distances from the surface listed above will only rarely escape back into the bulk without creating a Frenkel pair.

3. Clusters of three He atoms at greater initial distances from the surface take progressively longer times to reach the position for creation of a $\mathrm{He}_{3}$-vacancy complex. This is simply 
an increase of the diffusional time to reach the surface, which is proportional to distance to the surface squared.

4. A change of temperature does not produce any visible effect on the process, but increases the fraction of more energetic migration jumps relative to the rotation events. Since the interaction between the He cluster and the surfaces is rather weak, only the last couple of jumps towards the surface (before the cluster is immobilized) appear to differ from random walk. Only in the narrow region near the surface (see Fig. 4) does the energy gain by jumping towards the surface appear to be significant.

Although interstitial He clusters are highly mobile in the tungsten lattice, they are immobilized near a surface by their creation of a Frenkel pair. The resulting vacancy is incorporated into a He-vacancy cluster which is immobile over the accessible time scales for the temperatures of interest here. When this reaction occurs far from the free surface, the self-interstitial that has been created tends to be trapped near the He-vacancy cluster. However, in this case the interstitial tungsten atom moves to the surface in a $\langle 111\rangle$ direction, creating an adatom. The formation of immobile He-vacancy clusters should lead to a significant increase in He atom retention and the adatoms alter the surface morphology. Far from a surface, a larger number of He atoms is required in the cluster to activate creation of a Frenkel pair. Depending on the temperature, the minimum value is about six He atoms; an example is shown in Fig. 6 for the case of a cluster containing $10 \mathrm{He}$ atoms. After a near-surface He-vacancy complex is formed by Frenkel pair creation, its lack of mobility makes it a likely trapping site for additional He atoms. This will lead to the growth of an increasingly pressurized He-vacancy cluster which at some point will create another Frenkel pair to relieve the pressure. This process will provide a continuing source near-surface swelling and of surface modification due to a flux of adatoms.

\section{Discussion and comparison with other work}

The small defect complexes studied in the present work cannot be observed in experiments. The validity of the results obtained here, which do not include atomic vibrations, may be verified by comparing with concurrent MD simulations performed using the same set of interatomic potentials [11]. The authors analyzed 659 trajectories of a three He cluster migrating at $1200 \mathrm{~K}$ when the initial location was 10 atomic layers below a $\{100\}$ surface. They also observed the cluster of three He interstitials to create Frenkel pair leading to a $\mathrm{He}_{3}$-vacancy complex and a 
tungsten adatom on the surface. The probability of Frenkel pair formation depended on the distance from the surface, being 15.6 and $71.7 \%$ for the fifth and fourth atomic layer distances. The other event detected was Frenkel pair formation in the third atomic layer, with a probability of $12.7 \%$. This was followed by either complete or partial cluster dissociation and He desorption from the surface.

In our SEAKMC calculations we analyzed 50 trajectories for clusters beginning at different distances from the $\{100\}$ surface at $1200 \mathrm{~K}$, with similar statistics collected for $\{110\}$ and $\{111\}$ surfaces. The behavior was dominated by low energy $(\sim 0.01 \mathrm{eV})$ rotation events, so that the number of jump events was not large and the He cluster approached the free surface in only 31 out of the 50 cases. In these 31 cases, Frenkel pair formation occurred in the third layer from the surface. We did not observe partial or total dissociation of the cluster. A potential explanation for the difference in the behavior between the MD and SEAKMC results is the absence of atomic thermal vibrations in SEAKMC. As a result, physical gas pressure is not accounted for in the SEAKMC simulations, which may lead to an underestimate of the elastic interactions in the system. Otherwise, the two simulation approaches lead to quite similar predictions.

\section{Conclusions}

The results presented here are consistent with and complement those recently obtained using molecular dynamics simulations $[10,11]$. Taken together, these results provide a good understanding of the complex behavior of He defects in tungsten, particularly near free surfaces. The presence of a nearby free surface changes the energy landscape associated with small He interstitial clusters. A cluster of three interstitial He atoms forms a triangular defect in the tungsten lattice, with two He atoms near one face of the unit cell, and a third He atom near one of the perpendicular faces (Fig. 2). Depending on which surface is nearby, the triangle may be parallel to the surface or either a single $\mathrm{He}$ atom or a pair of $\mathrm{He}$ atoms may be closer to the surface.

1. The interaction of a cluster of three He interstitials with the $\{110\},\{100\}$ and $\{111\}$ free surfaces in tungsten is rather short-ranged (reaching only a few lattice parameters), and weak, with the maximum binding energy of $0.3 \mathrm{eV}$ at an distance from $\{110\}$ surface.

2. The He interstitial clusters are highly mobile in the bulk, but are immobilized when they approach the surface due to Frenkel pair formation creating an immobile He-vacancy 
complex with a tungsten interstitial. In contrast to the trapping which occurs in the bulk, in this case the interstitial moves to the surface in a $\langle 111\rangle$ direction from the original cluster, creating an adatom there.

3. Some differences between these results and MD simulations reported in [11] indicate that atomic vibrations may play a stronger role in the energy landscape near free surfaces, leading to the Monte Carlo model somewhat underestimating the interaction between $\mathrm{He}$ clusters and free surfaces.

The results also indicate that the creation of a Frenkel pair is much easier near the surface than in the bulk and this may enhance immobilization of He clusters in this region. This mechanism of cluster immobilization and adatom formation may provide a significant source of He retention in and surface degradation in tungsten divertors. Clearly, the ejection of one adatom to the surface does not result in significant reconstruction of the surface. However, the cumulative effect of many such events should be studied to understand its potential as a unit process that may contribute to fuzz formation. These He-vacancy clusters may also provide significant trapping sites for tritium and increase tritium retention.

\section{Acknowledgments}

This work was supported by the U.S. Department of Energy, Office of Fusion Energy Sciences and Office of Advanced Scientific Computing Research through the Scientific Discovery through Advanced Computing (SciDAC) project on Plasma-Surface Interactions, under Award No. DE-SC0008875. 


\section{References}

[1] ITER Physics Basis, Nucl. Fusion 39 (1999) 2137.

[2] D. Nishijima, M.Y. YeN. Ohno, S. Takamura, J. Nucl. Mater. 329-333 (2004) 1029-1033.

[3] M.J. Baldwin, R.P. Doerner, Nucl. Fusion 48 (2008) 035001.

[4] R. E. Stoller, S. I. Golubov, P. J. Kamenski, T. Seletskaia, and Yu. N. Osetsky, Philos. Mag. 90 (2010) 923-934.

[5] D. M. Stewart, Yu. N. Osetskiy, and R. E. Stoller, J. Nucl. Mater. 417 (2011) 1110-1114.

[6] C. Becquart, C. Domain, Nucl. Instrum. Methods Phys. Res. B 255 (2007) 23-26.

[7] C. Becquart, C. Domain, J. Nucl. Mater. 385 (2009) 223-227.

[8] N. Juslin, B.D. Wirth, J. Nucl. Mater. 438 (2013) 1221-1223.

[9] F. Sefta, N. Juslin, K.D. Hammond, B.D. Wirth, J. Nucl. Mater. 438 (2013) 493-496.

[10] F. Sefta, K.D. Hammond, N. Juslin, B.D. Wirth, Nucl. Fusion 53 (2013) 073015.

[11] L. Hu, K.D. Hammond, B.D. Wirth, D. Maroudas, Surf. Sci. 626 (2014) 21-25.

[12] N. Juslin, B.D. Wirth, J. Nucl. Mater. 432 (2013) 61-66.

[13] H. Xu, Y.N. Osetsky, R.E. Stoller, Phys. Rev. B 84 (2011) 132103.

[14] H. Xu, Y.N. Osetsky, R.E. Stoller, J. Phys.: Condens. Matter 24 (2012) 375402.

[15]. H. Xu, R.E. Stoller, Y.N. Osetsky, J. Nucl. Mater. 443 (2013) 66-70

[16] G. Henkelman, H. Jonsson. J. Chem. Phys. 111 (1999) 7010-7022.

[17] A.B. Bortz, M.H. Kalos, J.L. Lebowitz, J. Comput. Phys. 17 (1975) 10-18.

[18] H.X. Xu, R.E. Stoller, Y.N. Osetsky, D. Terentyev, Phys. Rev. Lett. 110 (2013) 265503.

[19] G.J. Ackland, R. Thetford, Philos. Mag. A 56 (1987) 15-30.

[20] D.E. Beck, Mol. Phys. 14 (1968) 311-315.

[21] K. Morishita, R. Sugano, B. Wirth, T. Diaz de La Rubia, Nucl. Instrum. Meth. Phys. Res. B 202 (2003) 76-81.

[22] P. Derlet, D. Nguyen-Manh, S. Dudarev, Phys. Rev. B 76 (2007) 054107. 


\section{Figure captions}

Fig. 1. Geometry for simulations: periodic boundary conditions along $x$ and $y$ directions, with a free surface perpendicular to $z$ direction. Initial positions of typical $\mathrm{He}_{3}$ interstitial clusters are shown with surface-to-cluster distances indicated; $\mathrm{a}_{\mathrm{o}}$ is the $\mathrm{W}$ lattice parameter.

Fig. 2. Configuration of a relaxed three-interstitial He cluster in a bcc tungsten unit cell with W atoms shown as large red spheres and He atoms as small green spheres.

Fig. 3. Binding energy between a three He atom interstitial cluster and a) $\{110\}$, b) $\{100\}$ and c) $\{111\}$ free surfaces.

Fig. 4. Saddle point (a) and system (b) energies as a function of distance between a three He atom interstitial cluster and the $\{100\}$ surface.

Fig. 5. Configuration of a $\mathrm{He}_{3}$-vacancy complex (three green spheres with blue sphere at center) and the corresponding tungsten adatom (red sphere) on the $\{100\}$ surface just after Frenkel pair formation. The tungsten adatom moves in the $\langle 111\rangle$ direction from the complex; the same result is observed for the other surfaces.

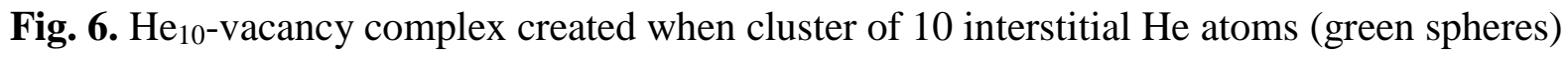
creates a Frenkel pair, location is far from any free surface. Created vacancy is shown as a blue sphere in the $\mathrm{He}_{10}$-vacancy complex and the corresponding $\langle 110\rangle$ dumbbell interstitial is shown as two red spheres centered on blue lattice site. 
Fig. 1. Geometry for simulations: periodic boundary conditions along $x$ and $y$ directions, with a free surface perpendicular to $z$ direction. Initial positions of typical $\mathrm{He}_{3}$ interstitial clusters are shown with surface-to-cluster distances indicated; $a_{o}$ is the W lattice parameter.

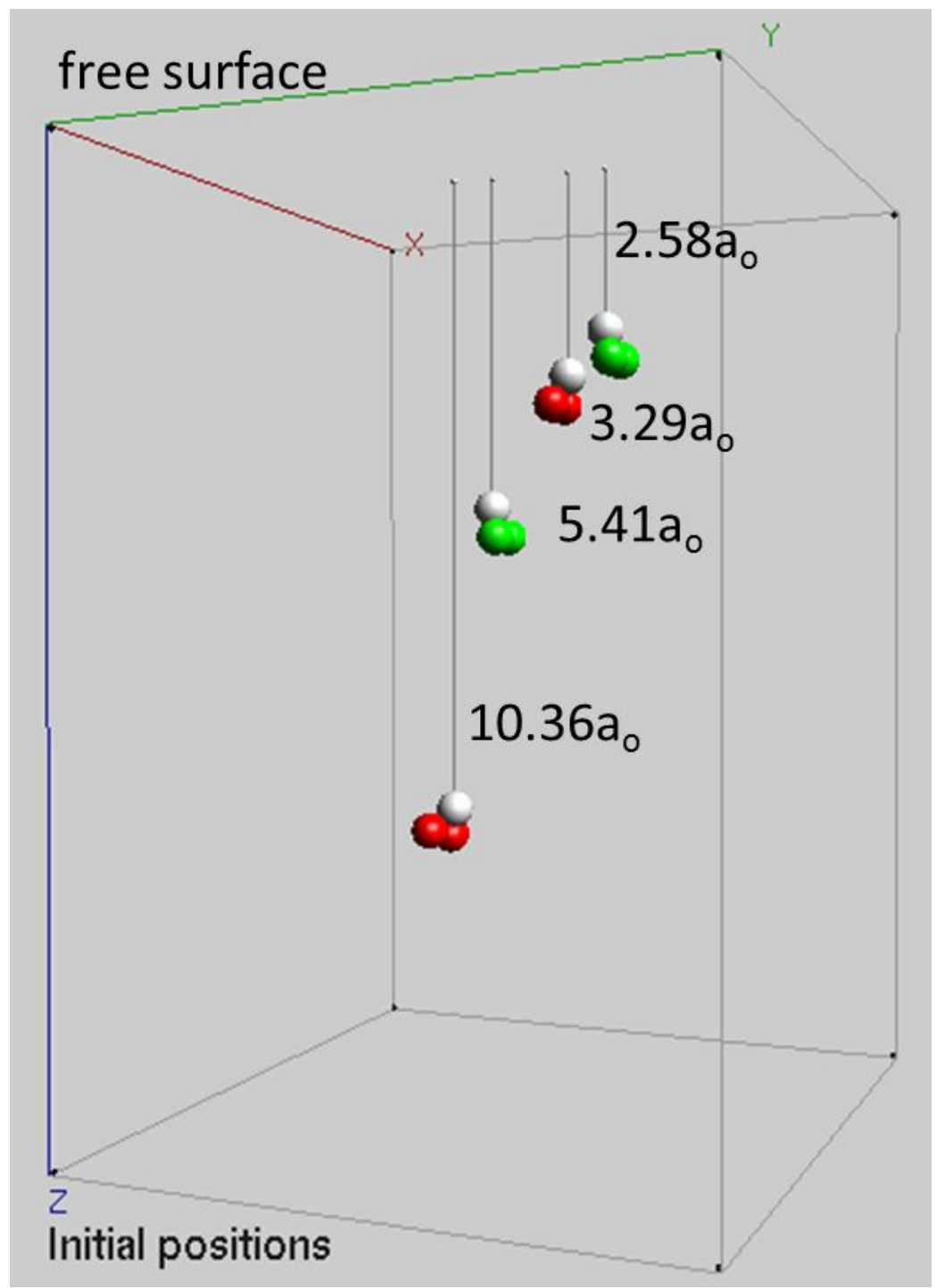


Fig. 2. Configuration of a relaxed three-interstitial He cluster in a bcc tungsten unit cell with W atoms shown as large red spheres and $\mathrm{He}$ atoms as small green spheres.

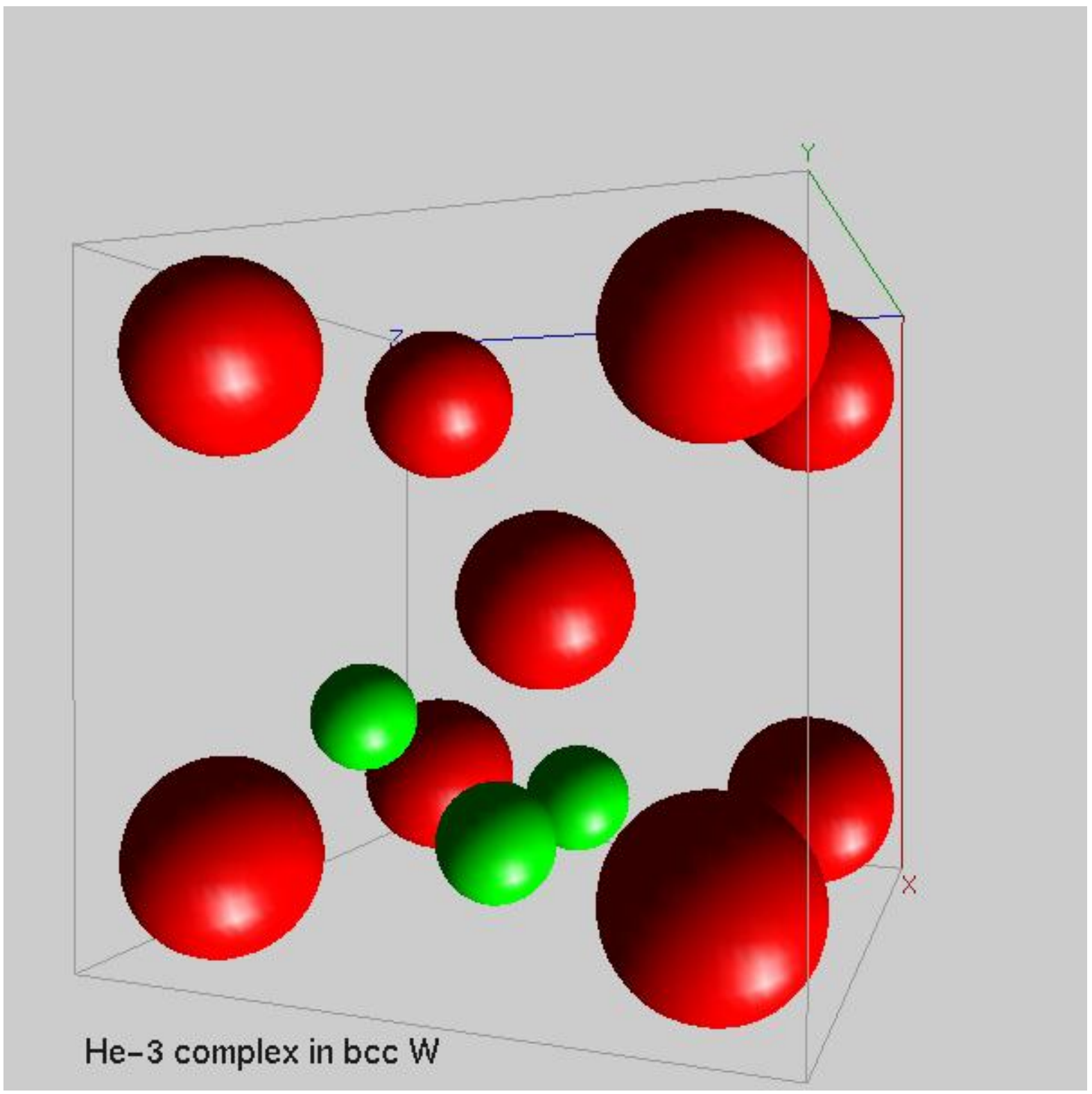


Fig. 3. Binding energy between a three He atom interstitial cluster and a) $\{110\}$, b) $\{100\}$ and c) $\{111\}$ free surfaces.
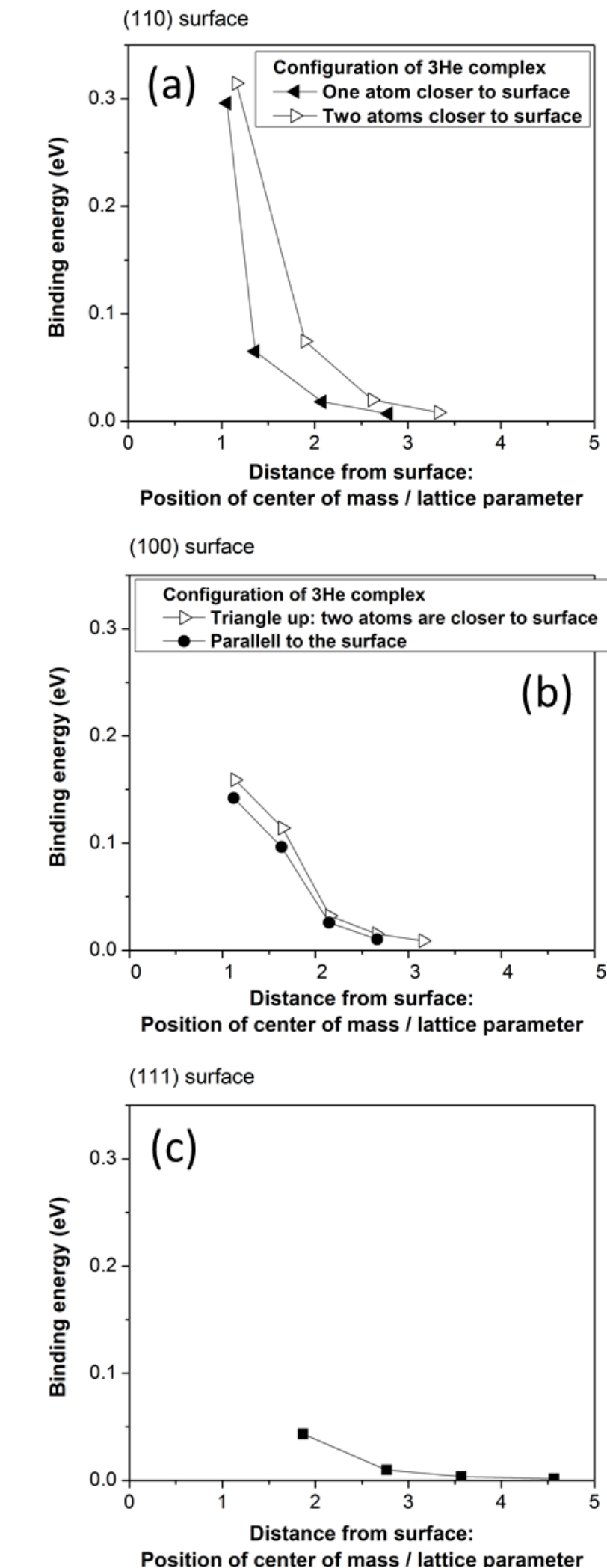
Fig. 4. Saddle point (a) and system (b) energies as a function of distance between a three He atom interstitial cluster and the $\{100\}$ surface.

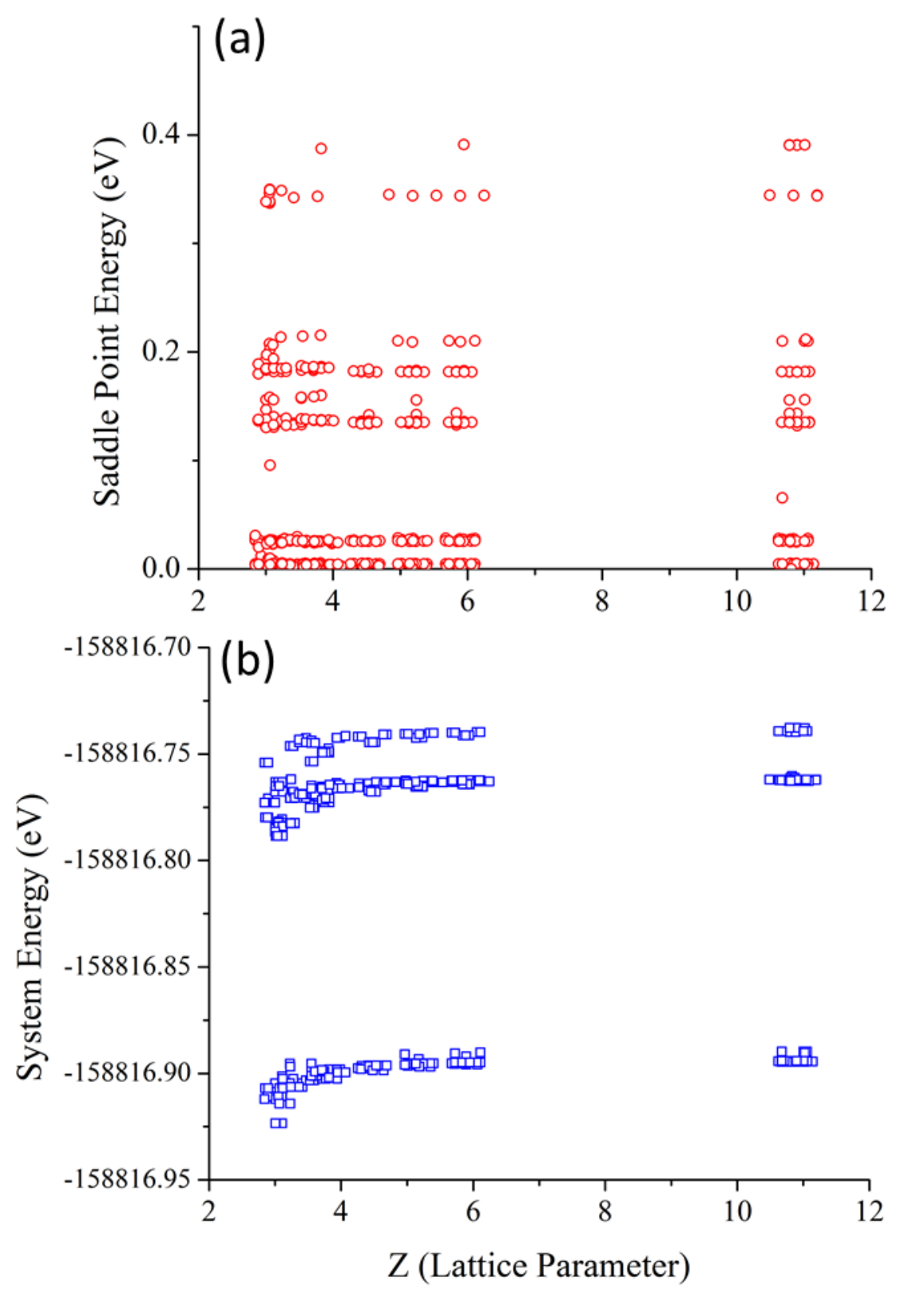


Fig. 5. Configuration of a $\mathrm{He}_{3}$-vacancy complex (three green spheres with blue sphere at center) and the corresponding tungsten adatom (red sphere) on the $\{100\}$ surface just after Frenkel pair formation. The tungsten adatom moves in the $\langle 111\rangle$ direction from the complex; the same result is observed for the other surfaces.

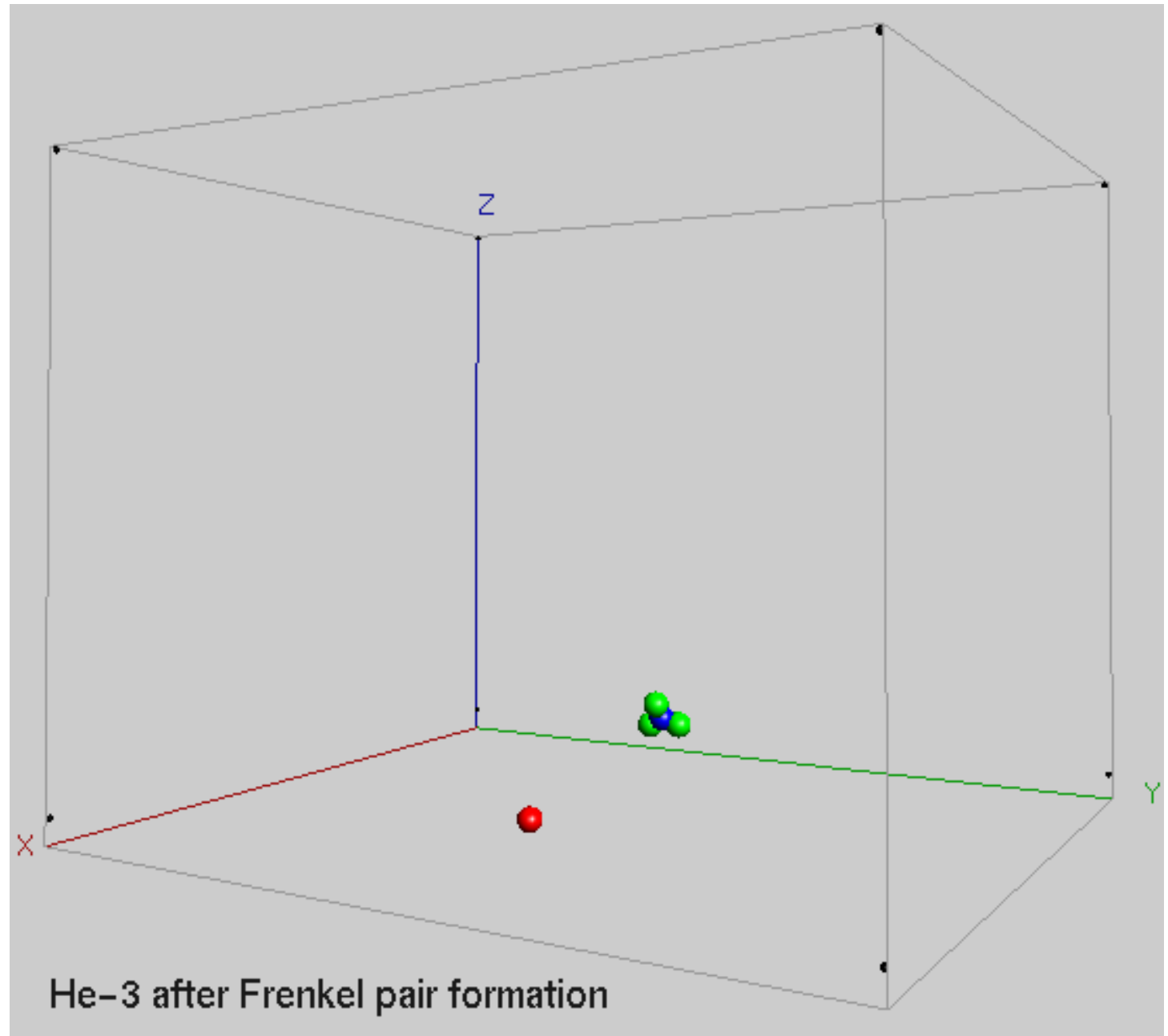


Fig. 6. $\mathrm{He}_{10}$-vacancy complex created when cluster of 10 interstitial He atoms (green spheres) creates a Frenkel pair, location is far from any free surface. Created vacancy is shown as a blue sphere in the $\mathrm{He}_{10}$-vacancy complex and the corresponding <110> dumbbell interstitial is shown as two red spheres centered on blue lattice site. 